\title{
UNITARITY AND FUNCTORIALITY
}

\author{
J. W. COGDELL AND I. I. PIATETSKI-SHAPIRO
}

Dedicated to M. Gromov on the occasion of his 50th birthday

Let $G$ be a reductive algebraic group defined over a global field $k$. Denote by $A(G)$ the set of all automorphic representations of $G(\mathbb{A})$. Denote by $A^{s}(G)$ the set of all unitary automorphic representations which occur in Langlands' spectral decomposition of $L^{2}(G(k) \backslash G(\mathbb{A}))$ and $A^{d}(G)$ those which occur discretely. Throughout this paper, cuspidal automorphic representations will mean unitary cuspidal automorphic representations.

Let $G$ and $H$ be split reductive groups over $k$. Assume that there exists a homomorphism of L-groups

$$
\rho:{ }^{L} H \rightarrow{ }^{L} G .
$$

According to Langlands' functoriality conjecture $\rho$ induces a map, which we denote by $\hat{\rho}$,

$$
\hat{\rho}: A(H) \rightarrow A(G) .
$$

If $\pi \in A(H)$ we will call its image under $\hat{\rho}$ a Langlands lift of $\pi$ corresponding to $\rho$. We also have compatible local versions $\hat{\rho}_{v}$ of this lifting. We will say the $\Pi \in A(G)$ is a weak Langlands lifting (or simply a weak lifting) of $\pi$ if for all places $v$ of $k$ where $\pi_{v}$ is unramified we have $\Pi_{v}$ is the local Langlands lifting of $\pi_{v}$. The difference between a weak lifting and Langlands lifting is that for weak lifting we do not require local compatibility at the archimedean places or at the finite ramified places.

In this paper we would like to investigate the behavior of $A^{s}(H)$ under Langlands liftings, in particular, to investigate conditions under which $\hat{\rho}(\pi) \subset A^{s}(G)$ for $\pi \in$ $A^{s}(H)$.

\section{Sufficient CONDITIONS}

We begin by giving sufficient conditions on $\pi \in A^{s}(H)$ to ensure that $\hat{\rho}(\pi) \subset$ $A^{s}(G)$. In light of its importance, as well as for simplicity of exposition, let us restrict to the case where the target group $G=G L(N)$.

Definition. Let $\pi$ be an automorphic representation of the group $H$. If $v$ is a place where the local component $\pi_{v}$ of $\pi$ is unramified, let $\lambda_{v, 1}, \ldots, \lambda_{v, n}$ denote the eigenvalues of the Satake conjugacy class in ${ }^{L} H$ associated to $\pi_{v}$. We will call $\pi$ weakly Ramanujan if

$$
\inf _{v} \max _{i} \frac{\left|\log \left(\left|\lambda_{v, i}\right|\right)\right|}{\log \left(q_{v}\right)}=0
$$

where the infimum is taken over all places at which $\pi_{v}$ is unramified.

The first author was supported in part by NSA grants MDA904-91-H-0040 and MDA904-93H-3028.

The second author was supported in part by NSF grants DMS-8807336 and DMS-9302732. 
If $\pi$ has a single place $v$ such that $\pi_{v}$ is unramified and tempered, then $\pi$ will be weakly Ramanujan since $\left|\lambda_{v, i}\right|=1$ for all $i$ at this place. In general, $\pi$ will be weakly Ramanujan iff for every $\varepsilon>0$ there is a place $v$ at which $\pi_{v}$ is unramified and the Satake eigenvalues for $\pi_{v}$ satisfy the estimate

$$
q_{v}^{-\varepsilon}<\left|\lambda_{v, i}\right|<q_{v}^{\varepsilon}
$$

for all $i=1, \ldots, n$. From the results of Jacquet and Shalika [2] it follows that any cuspidal automorphic representation of $G L(3)$ is weakly Ramanujan.

Theorem 1. Assume that $\pi \in A^{s}(H)$ is weakly Ramanujan. Then $\hat{\rho}(\pi)$ will be contained in $A^{s}(G L(N))$.

Proof. Let $\Pi \in \hat{\rho}(\pi)$ be a lifting (which we assume to exist). According to Langlands [4] there exist cuspidal automorphic representations $\sigma_{1}, \ldots, \sigma_{r}$ and real numbers $t_{1}, \ldots, t_{r}$ such that

$$
\Pi \subset \operatorname{Ind}_{P}^{G} \sigma_{1}|\operatorname{det}|^{t_{1}} \otimes \cdots \otimes \sigma_{r}|\operatorname{det}|^{t_{r}}
$$

where $P$ is a parabolic subgroup of $G=G L(N)$. The meaning of this formula is that $\Pi$ is a constituent of the induced representation. In order to prove our theorem it is enough to prove that $t_{1}=\cdots=t_{r}=0$.

For each place $v$ where the local component $\pi_{v}$ is unramified we let $\lambda_{v, 1}, \ldots, \lambda_{v, n}$ denote the eigenvalues of the Satake class associated to $\pi_{v}$. At these places $\Pi_{v}$ will also be unramified and we let $\Lambda_{v, 1}, \ldots, \Lambda_{v, N}$ denote the eigenvalues of the Satake class associated to $\Pi_{v}$. Since $\Pi_{v}$ is unramified at $v$ the local components $\sigma_{\ell, v}$ of the cuspidal representations $\sigma_{\ell}$ are also unramified and

$$
\Pi_{v} \subset \operatorname{Ind}_{P_{v}}^{G_{v}} \sigma_{1, v}|\operatorname{det}|^{t_{1}} \otimes \cdots \otimes \sigma_{r, v}|\operatorname{det}|^{t_{r}} .
$$

Let $\mu_{\ell, v, 1}, \ldots, \mu_{\ell, v, m_{\ell}}$ denote the Satake eigenvalues for $\sigma_{\ell, v}$.

Since $\pi$ is weakly Ramanujan for every $\varepsilon>0$ we have a place $v$ such that

$$
q_{v}^{-\varepsilon}<\left|\lambda_{v, i}\right|<q_{v}^{\varepsilon}
$$

for each $i=1, \ldots, n$. It is easy to see from finite dimensional representation theory that there will be a constant $c(\rho)$ depending only on $\rho$ such that

$$
q_{v}^{-c(\rho) \varepsilon}<\left|\Lambda_{v, j}\right|<q_{v}^{c(\rho) \varepsilon}
$$

for $j=1, \ldots, N$. Since each $\mu_{\ell, v, i} q_{v}^{-t_{\ell}}$ must be a $\Lambda_{v, j}$, then we have an estimate

$$
q_{v}^{-c(\rho) \varepsilon}<\left|\mu_{\ell, v_{m}, i} q_{v_{m}}^{-t_{\ell}}\right|<q_{v_{m}}^{c(\rho) \varepsilon} .
$$

Since each $\sigma_{\ell, v}$ is unitary, we know

$$
\left|\prod_{i=1}^{m_{\ell}} \mu_{\ell, v, i}\right|=\left|\omega_{\sigma_{\ell, n}}\left(\varpi_{v}\right)\right|=1
$$

$\omega_{\sigma_{\ell, v}}$ being the central character of $\sigma_{\ell, v}$. This gives us

$$
q_{v}^{-c(\rho) \varepsilon}<q_{v}^{t_{\ell}}<q_{v}^{c(\rho) \varepsilon}
$$

or

$$
-c(\rho) \varepsilon<t_{\ell}<c(\rho) \varepsilon
$$

for all $\ell=1, \ldots, r$. 
Since this is true for each $\varepsilon>0$ we obtain $t_{\ell}=0$ for $\ell=1, \ldots, r$.

Now we restrict our attention to $H=G L(n)$. Consider the Langlands lifting which corresponds to a homomorphism $\rho: G L(n, \mathbb{C}) \rightarrow G L(N, \mathbb{C})$. It is enough to consider the case when $\rho$ is irreducible. Our aim is to formulate sufficient conditions which imply that

$$
\hat{\rho}\left(A^{s}(G L(n))\right) \subset A^{s}(G L(N)) .
$$

Let us assume that any cuspidal automorphic representation of $G L(m), m \leq n$, is weakly Ramanujan.

Assume first that the representation $\pi \in A^{s}(G L(n))$ is generic. In this case we can get $\pi$ by unitary induction from cuspidal automorphic representations $\sigma_{1}, \ldots, \sigma_{m}$ of the linear groups $G L\left(n_{1}\right), \ldots, G L\left(n_{m}\right)$, where $n=n_{1}+\cdots+n_{m}$. According to the usual formalism of functoriality, to analyze the lift we have to restrict $\rho$ to

$$
G L\left(n_{1}, \mathbb{C}\right) \times \cdots \times G L\left(n_{m}, \mathbb{C}\right) \subset G L(n, \mathbb{C}) .
$$

Then $\rho$ becomes reducible and we have

$$
\rho=\bigoplus_{k} \rho_{1}^{k} \otimes \cdots \otimes \rho_{m}^{k}
$$

where $\rho_{i}^{k}$ will be a homomorphism of $G L\left(n_{i}, \mathbb{C}\right)$ into $G L\left(N_{i}^{k}, \mathbb{C}\right)$.

We now assume that for any $\rho_{i}^{k}$ there exists a corresponding Langlands lifting. Now we can apply Theorem 1 to each $\sigma_{i}$. From Theorem 1 we obtain that the Langlands lifting $\Sigma_{i}^{k}$ of $\sigma_{i}$ which corresponds to the homomorphism $\rho_{i}^{k}$ is lying in the spectral decomposition of $G L\left(N_{i}^{k}\right)$. Let $N^{(k)}=\prod N_{i}^{k}$ and let $\Sigma^{k}$ denote the Langlands lifting of $\Sigma_{1}^{k} \otimes \cdots \otimes \Sigma_{m}^{k}$ to $G L\left(N^{(k)}\right)$ associated to the natural embedding of L-groups $G L\left(N_{1}^{k}, \mathbb{C}\right) \otimes \cdots \otimes G L\left(N_{m}^{k}, \mathbb{C}\right) \hookrightarrow G L\left(N^{(k)}, \mathbb{C}\right)$. Arguing by Satake parameters as in the proof of Theorem 1 we see that $\Sigma^{k} \in A^{s}\left(G L\left(N^{(k)}\right)\right)$. Now we get $\Pi$ by unitary induction of the representation $\Sigma=\otimes_{k} \Sigma^{k}$ from the standard parabolic associated to the partition $N=\sum N^{(k)}$ to $G L(N)$. Thus $\Pi \in$ $A^{s}(G L(N))$.

We next consider the case when $\pi$ is not generic. It is clear that it is enough to consider the case when $\pi$ is a representation of the discrete spectrum but not cuspidal, since any representation lying in Langlands' spectrum decomposition can be gotten by unitary induction from representations of the discrete spectrum. In this case, we have to use the formalism which was suggested by J. Arthur [1]. Now we restrict $\rho$ to

$$
G L(m) \times \alpha_{\ell}(S L(2))
$$

where $n=m \ell$, and $\alpha_{\ell}$ is the irreducible $\ell$-dimensional representation of $S L(2)$. In order to explain how to attach $m$ and $\ell$ to a given representation $\pi$, we recall the following. It is possible to get any representation of the discrete spectrum of $G L(n)$ in the following way. Put $n=m \ell$. Denote by $\sigma$ a cuspidal automorphic representation of $G L(m)$. Then all the discrete non-cuspidal spectrum is obtained as the unique irreducible quotient of the induced representation $\operatorname{Ind}_{P}^{G L(n)} \sigma|\operatorname{det}|^{(\ell-1) / 2} \otimes \cdots \otimes \sigma|\operatorname{det}|^{-(\ell-1) / 2}$, where $P$ is the standard parabolic associated to the partition $n=m+\cdots+m$. We now put

$$
\rho\left(G L(m) \times \alpha_{\ell}(S L(2))\right)=\bigoplus_{j} \rho_{j} \otimes \alpha_{\ell_{j}}
$$

Now we can apply the result of Theorem 1 as in the generic case. 
Combining these two cases, we obtain the following theorem.

Theorem 2. Assume that every cuspidal representation of $G L(m), m \leq n$, is weakly Ramanujan and that all Langlands liftings from the various $G L\left(n_{i}\right)$ to $G L\left(N_{i}^{k}\right)$ needed above exist. Then for any homomorphism $\rho: G L(n, \mathbb{C}) \rightarrow G L(N, \mathbb{C})$ we have $\hat{\rho}\left(A^{s}(G L(n)) \subset A^{s}(G L(N))\right.$.

\section{An EXAMPLE}

If the target group $G$ is not a $G L(N)$ then it is no longer true that under Langlands lifting $A^{s}(H)$ necessarily goes into $A^{s}(G)$. In fact, there are cases where certain representations in $A^{s}(H)$ do not even have a weak Langlands lifts which lie in $A^{s}(G)$. The example presented below is the first example of this phenomenon.

Our example is the following. Put $H=S O(3) \times S O(3)$, and $G=S O(5)$, both groups split. Then ${ }^{L} H=S L(2, \mathbb{C}) \times S L(2, \mathbb{C})$, and ${ }^{L} G=S p(4, \mathbb{C})$. It is obvious that there exists an embedding

$$
\rho:{ }^{L} H \rightarrow{ }^{L} G .
$$

Denote by $\sigma$ a cuspidal automorphic representation of $P G L(2) \simeq S O(3)$ and by 1 the trivial representation. We will prove that if $L\left(\sigma \otimes \chi, \frac{1}{2}\right) \equiv 0$ for every quadratic character $\chi$, then $\hat{\rho}(\sigma \otimes \mathbf{1}) \notin A^{s}(G)$. In fact, we will prove $\sigma \otimes \mathbf{1}$ does not have any weak lifting in $A^{s}(G)$. It is not difficult to show, that if $L\left(\sigma \otimes \chi, \frac{1}{2}\right) \not \equiv 0$, then $\hat{\rho}(\sigma \otimes \mathbf{1}) \in A^{s}(G)$. Even more, $\hat{\rho}(\sigma \otimes \mathbf{1})$ is lying discretely in $L^{2}(G(k) \backslash G(\mathbb{A}))$. If $L\left(\sigma, \frac{1}{2}\right)=0$ and $L\left(\sigma \otimes \chi, \frac{1}{2}\right) \not \equiv 0$, then $\hat{\rho}(\sigma \otimes \mathbf{1})$ will be a cuspidal automorphic representation of $G$.

The existence of automorphic cuspidal representations $\sigma$ of $P G L(2)$, such that

$$
L\left(\sigma \otimes \chi, \frac{1}{2}\right) \equiv 0,
$$

where $\chi$ is an arbitrary quadratic character, was proved by Waldspurger [8]. He also proved that the image of such a representation is always 0 under the $\theta$ correspondence

$$
\widehat{S L(2)} \leftrightarrow P G L(2),
$$

for an arbitrary choice of additive character $\psi$. At the same time the image of the local correspondence will be always not trivial. The reason for this is that according to Waldspurger, if $L\left(\sigma \otimes \chi, \frac{1}{2}\right) \equiv 0$, then any local component $\sigma_{v}$ of $\sigma$ will be a principal series representation. Interesting examples of such representations over $\mathbb{Q}$ have been given by Rohrlich [7].

Theorem 3. If $\sigma$ is a cuspidal automorphic representation of $P G L(2)$ such that for all quadratic characters $\chi$ we have $L\left(\sigma \otimes \chi, \frac{1}{2}\right) \equiv 0$ then $\hat{\rho}(\sigma \otimes \mathbf{1}) \notin A^{s}(G)$. In fact, no weak Langlands lift of $\sigma \otimes \mathbf{1}$ can lie in $A^{s}(G)$.

Before we begin the proof of Theorem 3, let us recall the following idea [5]. Denote by $P$ the maximal parabolic subgroup of $G$ which has an abelian radical. Denote by $Q$ the maximal parabolic of $G$ with nonabelian radical.

Definition. Let $S$ be the abelian radical of $P$. Let $k_{v}$ be a local field, and let $(\pi, V)$ be a representation of $G_{v}$. Let $\psi_{T}$ be a nondegenerate character of $S_{v}$. We say that $(\pi, V)$ has the $U$-property (uniqueness) if, whenever a linear functional $\ell_{T}$ satisfies

$$
\ell_{T}(\pi(s) v)=\psi_{T}(s) \ell_{T}(v)
$$


( $s \in S_{v}, v \in V, T$ nondegenerate), then

$$
\ell(\pi(\delta) v)=\ell_{T}(v) \quad \text { for all } \delta \in O_{T}^{\circ},
$$

where $O_{T}^{\circ}$ is the connected component of stabilizer of character $\psi_{T}$ in Levi subgroup of $P_{v}$.

Proof. (Theorem 3) Let us assume that $\Pi$ is a weak Langlands lift of $\sigma \otimes \mathbf{1}$ which lies in $A^{s}(G)$. Put $\Pi=\bigotimes_{v} \Pi_{v}$, where $\Pi_{v}=\hat{\rho}\left(\sigma_{v} \otimes \mathbf{1}_{v}\right)$ if $\sigma_{v}$ is unramified. Then at these unramified places

$$
L\left(\Pi_{v}, s\right)=L\left(\sigma_{v}, s\right)\left(1-q_{v}^{-s-\frac{1}{2}}\right)^{-1}\left(1-q_{v}^{-s+\frac{1}{2}}\right)^{-1} .
$$

From [5] it follows that $\Pi_{v}$ has the $U$-property at these places.

Now we are going to prove that $\Pi$ has the global $U$-property. This means that if

$$
\varphi_{T}(g)=\int_{S(k) \backslash S(\mathbb{A})} \varphi(x g) \psi_{T}^{-1}(x) d x
$$

is a nondegenerate Fourier coefficient, then

$$
\varphi_{T}(\delta g)=\varphi_{T}(g) \quad \text { for all } \delta \in O_{T}^{\circ}(\mathbb{A}) .
$$

This follows immediately from weak approximation since we already know that (2) is true for all $\delta \in O_{T}^{\circ}(k)$, (because $\varphi$ is automorphic) and for all $\delta \in O_{T}^{\circ}\left(k_{v}\right)$, for almost all $v$ (all $v$ for which $\sigma_{v}$ is unramified). This implies [5] that $\Pi_{v}$ has the $U$-property for all $v$.

From the fact that $\Pi_{v}$ satisfies the $U$-property at all places and our assumption that $\Pi_{v}$ is unitary, we obtain that each $\Pi_{v}$ is a $\theta$-lifting from $\widetilde{S L(2)}$. Hence we get that

$$
\Pi=\otimes \theta_{v}\left(\nu_{v}\right),
$$

where $\theta_{v}$ means a local $\theta$-lifting. It remains to prove that there exists an automorphic representation $\nu$ of $\widetilde{S L(2)}$ such that $\Pi$ will be a global theta lifting of $\nu$. In order to prove this we will use the following interpretation of $\nu$. Let us recall that according to [5] the representation $\Pi$ remains irreducible after restriction to $Q(\mathbb{A})$ and it can be written in the form

$$
\Pi=\operatorname{Ind}_{D}^{Q} \nu \otimes \beta_{\psi}
$$

where $\beta_{\psi}$ is the Weil representation of the unipotent radical of $Q$, which is a Heisenberg group. This shows that we can choose $\nu$ to be an automorphic representation. ( $\Pi$ is an automorphic representation!) Hence $\Pi$ is lying in the image of the so-called Saito-Kurokawa lifting [6].

Let $\tau$ denote the theta lifting of $\nu$ to $P G L_{2}$. As was proved in [6] or [8] this implies that $L\left(\tau \otimes \chi, \frac{1}{2}\right) \neq 0$ for some choice of quadratic $\chi$. A consequence of the fact that $\Pi$ is the theta lift of $\nu$ is then that

$$
L^{S}(\Pi, s)=L^{S}(\tau, s) \zeta^{S}\left(s+\frac{1}{2}\right) \zeta^{S}\left(s-\frac{1}{2}\right)
$$

where $S$ is a sufficiently large finite set of places such that $S$ includes all archimedean places and $\Pi_{v}$ is unramified at all places outside of $S$. However, from the definition of $\Pi$ it follows that

$$
L^{S}(\Pi, s)=L^{S}(\sigma, s) \zeta^{S}\left(s+\frac{1}{2}\right) \zeta^{S}\left(s-\frac{1}{2}\right)
$$


By strong multiplicity one for $P G L_{2}$ this gives $\sigma=\tau$. This contradicts our assumption that $L\left(\sigma \otimes \chi, \frac{1}{2}\right) \equiv 0$.

Waldspurger proved that if $L\left(\sigma \otimes \chi, \frac{1}{2}\right) \equiv 0$ for any quadratic character $\chi$, then any local component $\sigma_{v}$ will be a principal series representation. Using this fact it is easy to check that representation $\hat{\rho}(\sigma \otimes 1)$ will be a constituent of some induced representation. It is not difficult to check that each component of this representation will be equal to a local $\theta$-lifting. Hence this representation will be abstractly unitarizable. It is also not difficult to check that there exists a lift of this representation to $G L(4)$, which will then be in $A^{s}(G L(4))$.

\section{Conjectures}

To end we would like to formulate the following conjectures.

Conjecture 1. Let $H$ and $G$ be reductive groups over a global field $k$ and assume that $G$ is split. Consider the weak Langlands lifting associated to a homomorphism $\rho:{ }^{L} H \rightarrow{ }^{L} G$. If $\pi \in A^{d}(H)$ then there always exists a weak lifting $\Pi \in A(G)$ which is abstractly unitarizable.

Conjecture 2. Let $H$ be a reductive group over a global field $k$ and let $G=G L(N)$. Consider the weak Langlands lifting associated to a homomorphism $\rho:{ }^{L} H \rightarrow{ }^{L} G$. Then for any $\pi \in A^{s}(H)$ there exists a weak lifting $\Pi \in A^{s}(G)$.

To establish the second conjecture it would of course be enough to prove it for $\pi \in A^{d}(H)$.

In order to explain why these conjectures are in terms of weak Langlands lifts rather than Langlands lifts, we consider the following example. Let $H=G S p(4)$ and $G=G L(4)$ over the field $k=\mathbb{Q}$. Consider the Langlands lifting associated to the natural embedding of $\mathrm{L}$-groups

$$
\rho:{ }^{L} H=G S p(4, \mathbb{C}) \rightarrow{ }^{L} G=G L(4, \mathbb{C}) .
$$

We will give an example of a $\pi \in A^{d}(H)$ such that any Langlands lift $\Pi^{L}$ of $\pi$ will not lie in $A^{s}(G)$ but there will be a weak lift $\Pi^{w} \in A^{s}(G)$.

For $\pi$ we take the cuspidal automorphic representation associated to a holomorphic Siegel modular form of level 1, of sufficiently high weight so that $\pi_{\infty}$ is discrete series and such that $\pi$ lies in the image of the Saito-Kurokawa lift. This is Kurokawa's original example [3]. Either from Kurokawa or as in the proof of Theorem 3, we have associated to $\pi$ a cuspidal representation $\tau$ of $P G L(2)$ which is again unramified at all finite places and such that

$$
L_{f}(\pi, s)=L_{f}(\tau, s) \zeta\left(s+\frac{1}{2}\right) \zeta\left(s-\frac{1}{2}\right)
$$

As a weak lifting of $\pi$ we may take

$$
\Pi^{w}=\operatorname{Ind}_{P_{2,2}}^{G} \tau \otimes \mathbf{1}
$$

where $P_{2,2}$ is the parabolic subgroup of $G L(4)$ with Levi $G L(2) \times G L(2)$ and we have put $\tau$ on the first $G L(2)$ and the trivial representation 1 on the second $G L(2)$. $\Pi^{w}$ then lies in $A^{s}(G)$.

Any Langlands lift $\Pi^{L}$ of $\pi$ must agree with $\Pi^{w}$ at all finite places, but $\Pi_{\infty}^{L}$ must be tempered by the Langlands classification over $\mathbb{R}$. Therefore we cannot have $\Pi_{\infty}^{L}=$ Ind $\tau_{\infty} \otimes \mathbf{1}_{\infty}$ since the later representation is not tempered. Therefore $\Pi^{L}$ cannot lie in $A^{s}(G)$ since we have strong multiplicity one in this space. 
In fact,

$$
\Pi_{\infty}^{L}=\operatorname{Ind}_{P_{2,2}}^{G} \tau_{\infty} \otimes S t_{\infty}
$$

where by $S t$ we mean the Steinberg representation of $P G L(2)$. Both of the representations $\Pi^{w}$ and $\Pi^{L}$ are constituents of

$$
\operatorname{Ind}_{P_{2,1,1}}^{G} \tau \otimes||^{1 / 2} \otimes||^{-1 / 2} \text {. }
$$

This shows that both of these representations are indeed automorphic. $\Pi^{w}$ lies as a subrepresentation of the space of automorphic forms while $\Pi^{L}$ is only a sub-quotient of the space of automorphic forms.

Let us introduce a notion of totally unitary representations for groups over local fields. Let $\pi_{v}$ a be representation of a group $G_{v}$ over the local field $k_{v}$. We will call $\pi_{v}$ totally unitary if it is unitary and remains unitary under any Langlands lifting to a split group. With this definition, Conjecture 1 would have the following corollary.

Corollary 1 (Conjecture 1). Let $G$ be a reductive group over a global field $k$ and let $\pi \in A^{d}(G)$. Then every local component $\pi_{v}$ of $\pi$ is totally unitary.

This Corollary greatly restricts the local components of elements of $A^{d}(G)$, and in particular of cuspidal representations.

Let us consider now the Arthur group [1]. For the unramified case, this is just the product $\mathbb{Z} \times S L(2, \mathbb{C})$. For the archimedean case it will be $W \times S L(2, \mathbb{C})$ where $W$ is the Weil group of the archimedean field. If we modify the Arthur group by replacing $S L(2, \mathbb{C})$ by $S U(2)$, then it is clear that representations of the Arthur group and representations of this modified group which you get by restriction will be the same. To unitary representations of the modified Arthur group correspond totally unitary representations of $G_{v}$, but not conversely. The correspondence between representations of $G_{v}$ and homomorphisms of the Arthur group into ${ }^{L} G_{v}$ is described in [1].

Conjecture 3. Let $G$ be a reductive group over a global field $k$. Let $\pi \in A^{d}(G)$. For each place $v$ such that $\pi_{v}$ is unramified we have an associated a unitary homomorphism of the modified Arthur group $\rho_{v}: \mathbb{Z} \times S U(2) \rightarrow{ }^{L} G_{v}$ such that each of the maps $\rho_{v}$ are of the same type, i.e., the restriction $\rho_{v}: S U(2) \rightarrow{ }^{L} G^{o}$ is independent of the place $v$.

Conjecture 3 is the correct generalization of the Ramanujan conjecture to arbitrary groups. It says that the deviation from the usual Ramanujan conjecture is uniform for all unramified components of a given cuspidal representation. Conjecture 3 follows from Conjecture 2 and the Ramanujan conjecture for $G L(n)$.

Acknowledgement. We would like to thank J. Arthur, P. Deligne, R. Howe, and $R$. Langlands for many fruitful discussions on this topic.

\section{REFERENCES}

[1] J. Arthur And S. Gelbart, Lectures on automorphic L-functions. L-functions and Arithmetic: Proceedings of the Durham Symposium, July, 1989, London Mathematical Society Lecture Notes 153, Cambridge University Press, Cambridge (1991), 1-59.

[2] H. JaCquet AND J. Shalika, On Euler products and the classification of automorphic representations. Am. J. Math. I: 103 (1981), 499-558; II: 103 (1981), 777-815.

[3] N. Kurokawa, Examples of eigenvalues of Hecke operators on Siegel cusp forms of degree two. Inv. Math. 49 (1978), 149-165. 
[4] R. Langlands, On the notion of an automorphic representation. Proc. Symp. Pure Math. 33, part 1 (1979), 203-207.

[5] I. Piatetski-Shapiro, Special automorphic forms on PGSp 4 . Arithmetic and Geometry, Vol. I, 309-325, Progress in Mathematics, Vol. 35, Birkhäuser, Basel, 1983.

[6] I. Piatetski-Shapiro, On the Saito-Kurokawa lifting Inv. Math. 71 (1983), 309-338.

[7] D. Rohrlich, Nonvanishing of $L$-functions and structure of Mordell-Weil groups. J. reine angew. Math. 417 (1991), 1-26.

[8] J.-L. Waldspurger, Correspondances de Shimura et Quaternions. Forum Math. 3 (1991), 219-307.

Department of Mathematics, Oklahoma State University, Stillwater, OK 74078

Department of Mathematics, Yale University, New Haven, CT 06520 